\title{
Obituary/Nécrologie
}

\section{Dr. John W. R. McIntyre 1925-1998}

"Quietly they go, the intelligent, the witty, the brave."

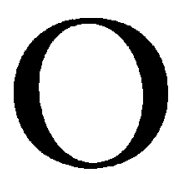

N March 12th, 1998, following a fatal road accident, the University of Alberta lost a fine scholar and an indefatigable teacher, who was also one of the campus's great characters and eccentrics - a man who made a unique contribution to the fabric of the University.

Dr John McIntyre had been associated with the University Hospital and the Faculty of Medicine for over forty years; he was well-known in other faculties and departments, a factor which allowed him to utilise extra-medical resources and expertise when setting up teaching programmes.

He was born in the United Kingdom in 1925, received his early education at Wellington School and completed his medical education at St Bartholomew's Hospital Medical School, London in 1948. Almost immediately he showed an interest in Anaesthesia, and after an initial residence, served as an anaesthetist in the Royal Army Medical Corps in Malaya, where he was attached to a field surgical team with the 48th Gurkha Infantry Brigade. In 1954 he became a Fellow of the Faculty of Anaesthetists at the Royal College of Surgeons, and in 1956 moved to Canada joining the active medical staff of the University of Alberta hospital, where he remained until his retirement in 1991. In 1974 John was appointed Professor of Anaesthesia at the University of Alberta, and on his retirement he became Professor Emeritus (Anaesthesia.)

During his career John was vigilant in promoting the interests of academic anaesthesia and this required great courage and discipline especially in the early years when he was almost alone in trying to promote those interests in a department which had a heavy commitment to clinical service. The writer remembers with gratitude the encouragement and advice received from him in the late ' 60 's while preparing a scientific paper. In those days persuading a resident to take time out from clinical studies to undertake research was a formidable task, but somehow John seemed to manage it. He was a great friend to the resident-in-training, always prepared to go the extra mile.
His scholarly activities continued throughout his working life and into retirement. He published numerous original articles, chapters, books and letters. Special areas of interest included securement and management of the airway, and monitoring during anaesthesia. For many years he worked as Executive Director and/or member of the Test Committee in Anaesthesia at the R S McLaughlin Examination and Resource Centre, and thus he was intimately involved in the development of the multiple-choice question in Anaesthesia specialty examinations in Canada. For twenty years he was a reviewer for the Canadian Anaesthetists' Society Journal, and was an editorial board member of the International Journal of Clinical Monitoring and Computing (1984 - 1997). In the 1970 's, when the West began to take an interest in traditional Chinese medicine John served on the two allCanadian medical delegations to the People's Republic of China. Members of academic departments across North America and around the world knew and respected his abilities and energy; he held visiting Professorships in China, Japan, Russia, The Netherlands and India, presenting many guest lectures at international meetings.

John's interests did not flag in retirement, in fact they seemed to take on a new strength when as Professor Emeritus he developed a close relationship with the University of Hirosaki in Japan, receiving a Distinguished Alumnus Medal from that institution in 1995. His most recent book "Tracheal Intubation" was published in Japan in 1998. He was also involved in teaching Japanese colleagues how to write and present scientific articles for English language journals.

John McIntyre was a generous and kind man with an eclectic mind. He had a great skill as a raconteur, and a robust humour which leavened his wisdom, making him a convivial companion. A prodigious reader, he could be discovered looking into such diverse subjects as the origin of the joke, ceramics and porcelains, and the architecture of the English workhouse. Music was a life-long passion, and the Edmonton Symphony Orchestra benefited from his generosity. He was an inveterate walker and pursued 
this activity in many interesting parts of the world.

In 1990 the Department of Anaesthesia Library at the University of Alberta Hospital was named for Dr John W.R. McIntyre, a fitting tribute to a singular person who devoted his life to the University of Alberta and academic medicine. His many colleagues, students and friends will not easily forget him.

\section{Donald Dobson \\ 19 March 1998}

"Ils s'en vont doucement, les êtres intelligents, vifs, courageux".

Le 12 mars 1998, à la suite d'un fatal accident de la route, l'Université de l'Alberta perdait un érudit raffiné et un professeur infatigable dont le non-conformisme en faisait également un des grands personnages du campus - un homme qui a contribué de façon unique à la structure de l'université.

Le docteur John McIntyre a été rattaché à l'Hôpital universitaire et à la Faculté de médecine pendant plus de quarante ans; on le connaissait bien dans les autres facultés et départements, ce qui lui permettait d'utiliser des connaissances et des ressources extramédicales lors de la composition de programmes d'enseignement.

Il est né au Royaume-Uni en 1925, a d'abord fréquenté l'école Wellington et a terminé ses études médicales à l'école de médecine de l'hôpital St Bartholomew à Londres, en 1948. Il a manifesté presque aussitôt son intérêt pour l'anesthésie et, après une résidence initiale, est entré au service de santé de l'armée en Malaisie en qualité d'anesthésiste et a été affecté à une équipe chirurgicale de campagne avec la $48^{\mathrm{c}}$ Brigade d'infanterie de Gurkha. En 1954, il est devenu membre de la faculté des anesthésistes du Collège royal des chirurgiens et, en 1956, il est venu au Canada et s'est joint au personnel médical actif de l'hôpital de l'Université de l'Alberta où il est resté jusqu'à sa retraite en 1991. En 1974, John a été nommé professeur d'anesthésie à l'Université de l'Alberta, puis professeur émérite (anesthésie) à sa retraite.

Au cours de sa carrière, John a été attentif à promouvoir les intérêts de la recherche en anesthésie, ce qui a exigé beaucoup de courage et de discipline pendant les premières années alors qu'il était pratiquement le seul à oser faire une telle promotion dans un département qui était très engagé dans le service clinique. L'auteur se rappelle avec gratitude l'encouragement et les conseils qu'il en a reçus à la fin des années 60 lors de la préparation d'un article scientifique. À cette époque, persuader un résident de consacrer une partie de son temps d'étude clinique à l'élaboration d'une recherche était une formidable tâche mais, d'une façon ou d'une autre, il semble que John y soit parvenu. C'était un grand ami des étudiants en résidence et il était toujours prêt à aller de l'avant.

Il a poursuivi ses activités de recherche tout au long de sa carrière et même à sa retraite. Il a publié de nombreux articles originaux, des chapitres, des livres et des lettres. Ses principaux centres d'intérêt concernaient la sécurisation et la prise en charge de l'intubation ainsi que la surveillance pendant l'anesthésie. Pendant de nombreuses années, il a travaillé comme directeur ou membre, ou les deux, du Comité d'examen en anesthésie au Centre d'étude et de documentation $\mathrm{R}$ $S$ McLaughlin et, de ce fait, a été associé de près au développement de questions à choix multiples des examens spécialisés en anesthésie au Canada. Pendant vingt ans, il a été réviseur pour le Journal de la société canadienne des anesthésistes et membre de l'équipe de direction de l'International Journal of Clinical Monitoring and Computing (1984-1997). Dans les années 70 , quand l'Occident a commencé à s'intéresser à la médecine chinoise traditionnelle, John a fait partie des deux délégations médicales entièrement canadiennes en République populaire de Chine. Les membres de départements de recherches à travers l'Amérique du Nord et dans le monde connaissaient et respectaient son talent et son énergie; il a été professeur associé en Chine, au Japon, en Russie, aux Pays-Bas et en Inde, il était souvent le conférencier invité aux rencontres internationales.

Les intérêts de John n'ont pas diminué à la retraite. De fait, ils ont connu une nouvelle vigueur semble-til quand, à titre de professeur émérite John a développé une relation étroite avec l'Université de Hirosaki au Japon de qui il a reçu une médaille de distinction des anciens en 1995. Son livre le plus récent, Tracheal Intu bation, a été publié au Japon en 1998. Il a aussi enseigné à des collègues japonais comment rédiger et présenter des articles scientifiques dans des journaux de langue anglaise.

John McIntyre était un homme bon et généreux à l'esprit éclectique. Ses grandes qualités de conteur et son grand sens de l'humour contrastaient avec sa sagesse, faisant de lui un joyeux drille. Lecteur insatiable, on pouvait le trouver en train de chercher des sujets aussi différents que l'origine des plaisanteries, les céramiques et les porcelaines, l'architecture des hospices anglais. La musique a toujours été une passion pour lui et l'orchestre symphonique d'Edmonton a bénéficié de sa générosité. Il était un marcheur infatigable et il a pratiqué cette activité dans beaucoup d'endroits intéressants dans le monde.

En 1990, la bibliothèque d'anesthésie de l'Hôpital de I'Université de l'Alberta a reçu le nom du Dr John W.R 
McIntyre, un hommage mérité rendu à une personne unique qui a voué sa vie à l'Université de l'Alberta et à la recherche médicale. Ses nombreux collègues, étudiants et amis ne l'oublieront pas facilement.

Donald Dobson

19 mars 1998 